\title{
Psychosocial Determinants of Quality of Life 6 Months After Transplantation: Longitudinal Prospective Study
}

\author{
D. Telles-Correia, A. Barbosa, I. Mega, E. Mateus, and E. Monteiro
}

\begin{abstract}
Objectives. We sought to investigate the psychosocial determinants of quality of life at 6 months after transplantation.

Methods. A sample of liver transplant candidates $(n=60)$, composed of consecutive patients (25\% with familial amyloid polyneuropathy [FAP]) attending outpatient clinics was assessed in the pretransplant period using the Neo Five Factor Inventory, Hospital Anxiety and depression Scale (HADS), Brief COPE, and SF-36, a qualityof-life, self-rating questionnaire. Six months after transplantation, these patients were assessed by means of the SF-36.
\end{abstract}

Results. Psychosocial predictors where found by means of multiple regression analysis. The physical component of quality of life at 6 months after transplantation was determined based upon coping strategies and physical quality of life in the pretransplant period (this model explained $32 \%$ of variance). The mental component at 6 months after transplantation was determined by depression in the pretransplant period and by clinical diagnoses of patients. Because FAP patients show a lower mental component of quality of life, this diagnosis explained $25 \%$ of the variance.

Conclusions. Our findings suggested that coping strategies and depression measured in the pretransplant period are important determinants of quality of life at 6 months after liver transplantation.

$\mathrm{O}$ VER THE LAST decade, liver transplantation has proven to be the treatment of choice for patients with terminal liver disease. It has been demonstrated that quality of life can be improved after transplantation in liver disease patients. ${ }^{1-4}$ Previous investigations into quality of life among transplant recipients have primarily focused on the relation between medical treatment, course of transplantation (revisions, retransplantation, rejection), and patient quality of life. Some authors have observed that maladaptive coping strategies and the presence of high levels of anxiety/depression after transplantation can relate to poor outcomes and a poorer quality of life.$^{5-7}$ Nevertheless, the extent to which anxiety, depression, and coping strategies present in the pretransplant period have not yet been well established to affect physical and mental quality of life after liver transplantation. This study sought to investigate the psychosocial determinants (assessed before transplantation) of quality of life transplantation at 6 months after liver transplantations.

\section{METHODS}

Participants

We studied a set of 60 transplant candidates who were consecutive patients with paramyloidosis, alcoholic cirrhosis, and other liver diseases, attending weekly outpatient clinics and after informed consent agreeding to participating in our study between March 1, 2006 and March 1, 2007. The study protocol was approved by the institutional review committee (according to ethical guidelines of the 1975 Declaration of Helsinki). We assessed these patients before and 6 months after transplantation.

From the Psychiatric Approach to Liver Transplanted Patients' Unit, Curry Cabral Hospital's Liver Transplantation Center; and the Lisbon Faculty of Medicine, Lisbon, Portugal.

Address reprint requests to $\mathrm{Dr}$ Diogo Telles-Correia, Rua Sacadura Cabral $n^{\circ}$ 55, $4^{\circ}$ B, 1495 Cruz Quebrada-Dafundo, Portugal. E-mail: tellesdiogo@gmail.com

(c) 2009 by Elsevier Inc. All rights reserved. 360 Park Avenue South, New York, NY 10010-1710 
Table 1. Medical Diagnosis-Subgroups

\begin{tabular}{lc}
\hline \multicolumn{1}{c}{ Medical Diagnosis } & $n$ \\
\hline Familial amyloid polyneuropathy & 15 \\
Chronic liver diseases & 13 \\
Alcohol liver disease & 7 \\
Hepatitis C-associated cirrhosis & 2 \\
Hepatocellular carcinoma & 2 \\
Primary biliary cirrhosis & 1 \\
Familial progressive cholestasis & 18 \\
Mixed diagnosis & 2 \\
Unknown cause & \\
\hline
\end{tabular}

Note: A total of 60 patients were included in this review.

\section{Measurement of Quality of Life}

We used the Short Form (SF)-36 (Portuguese-validated version), a self-rating questionnaire developed by the Medical Outcome Trust, ${ }^{8}$ to investigate certain primary aspects of quality of life. The SF-36 has been widely shown under a range of medical conditions to have adequate reliability and validity. The first 4 subscales refer to physical aspects; the last 4 scales, mental aspects: physical functioning, physical role limitation, bodily pain general health, vitality, social functioning, emotional role limitation, and emotional well-being. Physical aspects mostly refered to physical capability to perform normal daily activities. The mental dimension mostly refers to social aspects of life and the degree to which illness interfered with emotional well-being and social roles. Total values were computed for physical and mental components of healthrelated quality of life by averaging the 8 weighted subscales using the coefficients generated by Hays et $\mathrm{al}^{9}$ in the Medical Outcomes Study.

We measured quality of life at 3 times: before and at 1 and 6 months after transplantation.

\section{Psychiatric and Psychological Evaluation}

Level of anxiety and depression were assessed by means of Hospital Anxiety and Depression Scale (HADS) ${ }^{10}$ which measures anxiety and depression in physically ill patients in particular. A total score of 11 or higher on each scale is regarded as indicative of a clinical diagnosis of anxiety or depression; a total score in the range of 8 to 10 is borderline; and values of 8 or lower are interpreted as clinically insignificant or normal. ${ }^{10}$ This instrument was validated to the Portuguese population by Pais-Ribeiro et al. ${ }^{11}$

Personality was assessed by means of NEO Five-Factor Inventory (NEO- FFI), which is a shortened version of the NEO PI-R. ${ }^{12}$ It has been validated for Portuguese by Bertoquini et $\mathrm{a}^{13}$ and designed to provide quick, reliable, and valid measures of the 5 domains of adult personality; openness to experience, conscientiousness, extroversion, agreeableness, and neuroticism. The 60 items are rated on a 5-point scale from 1 ("I completely disagree") to 5 ("I completely agree").

Coping strategies were assessed by means of the Brief COPE, a shortened version of COPE ${ }^{14}$ which was validated to the Portuguese population ${ }^{15}$ and designed to give a quick, reliable, and valid measures of the 14 domains of coping strategies: self-distraction, active coping, denial, substance use, use of emotional support, use of instrumental support, behavioural disengagement, venting, positive reframing, planning, humor, acceptance, religion, and selfblame. Responses were rated on a 4-point scale ranging from 1 ("I haven't been doing this at all") to 4 ("I've been doing this a lot").

\section{Statistical Methods}

Statistical analyses were performed using the SPSS 13.0 for Windows software package. Descriptive data were presented in absolute frequencies/percentages. Quality of life determinants were assessed by means of multiple linear regression.

\section{RESULTS}

\section{Demographic and Medical Data}

Regarding medical diagnoses, 15 patients had familial amyloid polyneuropathy (FAP) and 45 chronic liver diseases (CLD). Among the patients with chronic liver disease, 13 had alcoholic liver disease (ALD); 7, hepatitis C associated cirrhosis (HCAC); 2, hepatocellular carcinoma (HCC); 2, primary biliary cirrhosis; 1 , familial progressive cholestasis; 2 unknown cause and the others, mixed diagnoses: 7 ALD $+\mathrm{HCC} ; 7$ $\mathrm{HCAC}+\mathrm{HCC} ; 2 \mathrm{HCAC}+\mathrm{HCC}+\mathrm{ALD} ; 1 \mathrm{HCC}+$ hepatitis virus B liver disease; 1 hemochromatosis + LC (Table 1).

\section{Quality-of-Life Determinants}

We calculated Spearmen correlation coefficients between quality of life (mental and physical components, separately) and other variables: anxiety and depression (HADS); coping mechanisms (BRIEF COPE); personality dimensions (NEO-FFI); quality of life (mental and physical components); and medical diagnosis (FAP/liver disease). A multiple regression model was estimated using as dependent variable quality of life (mental and physical components, separately) and as independent ones the other correlated variables (obtained by Spearmen correlation coefficients). The medical diagnosis (FAP/liver disease) was also included as an independent variable. By means of the multiple linear regression model, we observed that the physical component of quality of life at 6 months after transplantation was determined by coping strategies and physical quality of life in the pretransplant period. This model explained $32 \%$ of the variance (Table 2). The mental component at 6 months after transplantation was determined by depression in the pretransplant period and by the clinical diagnosis of patients, namely, FAP patients with a lower mental component of quality of life explained $25 \%$ of the variance (Table 3 ).

\section{DISCUSSION}

Previous investigations into quality of life among transplant recipients have focused primarily on the relation to medical issues. Some authors have also found that maladaptive

Table 2. Physical Components of Quality-of-Life Determinants

\begin{tabular}{|c|c|c|}
\hline \multirow{2}{*}{$\begin{array}{l}\text { Psychiatric/psychosocial Variables in } \\
\text { Pretransplantation Period }\end{array}$} & \multicolumn{2}{|c|}{$\begin{array}{l}r=.571 ; r^{2}=.326 \\
\text { Adjusted } r^{2}=.299\end{array}$} \\
\hline & Beta & $t$ \\
\hline $\begin{array}{l}\text { Coping Strategies (Brief COPE): } \\
\text { Behavior desingagement }\end{array}$ & -3.02 & $-2.369^{\star}$ \\
\hline Quality of life: physical component & 0.283 & $3.408^{\star *}$ \\
\hline
\end{tabular}


Table 3. Mental Component of Quality-of-Life Determinants

\begin{tabular}{|c|c|c|}
\hline \multirow{2}{*}{$\begin{array}{c}\text { Psychiatric/psychosocial Variables in } \\
\text { Pretransplant Period }\end{array}$} & \multicolumn{2}{|c|}{$\begin{array}{l}r=.431 ; r^{2}=.25 \\
\text { Adjusted } r^{2}=.20\end{array}$} \\
\hline & Beta & $t$ \\
\hline Anxiety/depression (HADS): depression & -3.40 & $-2.60^{\star *}$ \\
\hline $\begin{array}{l}\text { Clinical diagnosis: FAP diagnosis } \\
\quad(1, \text { yes } / 2, \text { No) }\end{array}$ & 13.86 & $2.30^{\star}$ \\
\hline
\end{tabular}

coping strategies and the presence of high levels of anxiety/ depression after transplantation can be related to poor outcomes and a poor quality of life.

We think that it is important to identify maladaptive psychological characteristics pretransplantation; they can influence quality of life after transplantation. Strategies must be designed to reduce the risk of such characteristics. This kind of information can also be useful to select candidates before liver transplantation.

Our findings suggested that coping strategies and depression measured in the pretransplant period are important determinants of quality of life at 6 months after liver transplantation. Causal relations were observed since this was a prospective study.

Further studies are needed to identify the extent to which early, continued, psychotherapeutic interventions improve not only mental but also physical quality of life among patients at risk; for example, patients with increased scores in the pretransplant period for depression or for maladaptive coping strategies.

\section{REFERENCES}

1. Tarter RE, Switala J, Arria A, et al: Quality of Life before and after orthopic hepatic transplantation. Arch Intern Med 151:1521, 1991
2. Moore KA, Jones R, Angus MCL, et al: Psychosocial adjustment to illness: quality of life following liver transplantation. Transplant Proc 24:2257, 1992

3. Riether AM, Smith SL, Lewison BJ, et al: Quality of life changes and psychiatric and neurocognitive outcome after heart and liver transplantation. Transplantation 54:444, 1992

4. Price CE, Lowe D, Cohen AT, et al: Prospective study of the quality of life in patients assessed for liver transplantation: outcome in transplanted and not transplanted groups. J R Soc Med 88:130, 1995

5. Zhang SJ, Huang LH, Wen YL, et al: Impact of personality and coping mechanisms on health-related quality of life in liver transplantation recipients. Hepatobil Pancreat Dis Int 4:356, 2005

6. Myakovsky L, Dew MA, Switzer GE: Avoidant coping with health problems is related to poorer quality of life among lung transplant candidates. Prog Transplant 13:183, 2003

7. Nickel R, Wunsch A, Egle UT, et al: The relevance of anxiety, depression, and coping in patients after liver transplantation. Liver Transpl 8:63, 2002

8. Ware J: SF-36 Health Survey: Manual and interpretation guide. Boston: Nimrod; 1993

9. Development of the Portuguese version of MOS SF-36. Part II-validation tests. Acta Med Port 13:119, 2000

10. Zigmond AS, Snaith AP: The Hospital Anxiety Depression Scale. Acta Psych Scand 67:361, 1983

11. Pais-Ribeiro J, Silva I, Ferreira T, et al: Validation study of a Portuguese version of the hospital anxiety and depression scale. Psychol Health Med 12:225, 2007

12. Costa PT Jr, McCrae RR: NEO PI-R professional manual. Odessa, Fla: Psychological Assessment Resources, Inc; 1992

13. Bertoquini V, Pais-Ribeiro JL: Estudo de Formas reduzidas do NEO-PI-R. Psicologia: Teoria, Investigação e Prática 85:102, 2006

14. Carver C: You want to measure coping but your protocol's too long. Consider the brief COPE. Int J Behav Med 92:100, 1997

15. Pais Ribeiro J, Rodrigues A: Questioning coping: a propos of the adaptation study of the Brief COPE. Psicologia: Saúde \& Doença; 2004, p 5 